\title{
High-conducting magnetic nanowires obtained from uniform titanium-covered carbon nanotubes
}

\author{
S. Dag, E. Durgun, and S. Ciraci \\ Department of Physics, Bilkent University, Ankara 06800, Turkey
}

(Received 10 November 2003; published 22 March 2004)

\begin{abstract}
We have shown that a semiconducting single-wall carbon nanotube (SWNT) can be covered uniformly by titanium atoms and form a complex but regular atomic structure. The circular cross section changes to a squarelike form, and the system becomes metallic with high state density at the Fermi level and with high quantum ballistic conductance. Metallicity is induced not only by the metal-metal coupling, but also by the band-gap closing of SWNT's at the corners of the square. Even more interesting is that uniform titaniumcovered tubes have magnetic ground state with significant net magnetic moment. Our results have been obtained by the first-principles pseudopotential plane-wave calculations within the density-functional theory.
\end{abstract}

DOI: 10.1103/PhysRevB.69.121407

The fabrication of interconnects with high conductance and low energy dissipation has been a real challenge in the rapidly developing field of nanoelectronics. Very thin metal wires and atomic chains have been produced by retracting the scanning-tunneling-microscope tip from an indentation and then by thinning the neck of the materials that wets the tip. ${ }^{1-3}$ While those nanowires produced so far played a crucial role in understanding the quantum effects in electronic and thermal conductance, ${ }^{4-6}$ they were neither stable nor reproducible to offer any relevant application. It has been shown that single-wall carbon nanotubes (SWNT's) can serve as templates to produce reproducible, very thin metallic wires with controllable sizes. ${ }^{7}$ Continuous Ti coating of varying thickness, and quasicontinuous coatings of $\mathrm{Ni}$ and $\mathrm{Pd}$ were obtained by using electron beam evaporation techniques. ${ }^{8}$ Good conductors, such as $\mathrm{Au}, \mathrm{Al}$, were able to form only isolated discrete particles or clusters instead of a continuous coating of SWNT's. Low-resistance Ohmic contacts to metallic and semiconducting SWNT's have been achieved also by $\mathrm{Ti}$ and $\mathrm{Ni}$ atoms. ${ }^{9}$ In spite of the impact made by these experimental works ${ }^{7-9}$ there has been very little effort so far to present an atomic scale understanding of uniform Ti coverage.

In this paper we show that a semiconducting s-SWNT is transformed to a good conductor as a result of Ti coverage. Moreover, Ti-covered tubes have magnetic ground state with a net magnetic moment. These results have important implications in nanoscience and nanotechnology. We carried out first-principles, spin-unpolarized (SU) and spin-relaxed (SR) calculations within the generalized gradient approximation. ${ }^{10}$ Calculations have been performed in momentum space by using periodically repeating tetragonal supercell with lattice constants, $a_{s}=b_{s} \sim 20 \AA$ and $c_{s}=c \quad[c$ being the onedimensional (1D) lattice constant of the SWNT]. The Brillouin zone of the supercell is sampled by using special k-point scheme. All atomic positions (i.e., all adsorbed Ti atoms and carbon atoms of the SWNT), as well as $c_{s}$ (hence c) have been optimized. Using same parameters of calculations we achieved to reproduce structural parameters of bulk $\mathrm{Ti}$, as well as $\mathrm{Ti}_{2}$ and $\mathrm{Ti}_{3}$ molecules.

The $(8,0)$ zigzag tube is a semiconductor, the band gap of which has been calculated to be $E_{g}=0.6 \mathrm{eV}$. An individual $\mathrm{Ti}$ atom is adsorbed at specific sites on the external and internal surfaces of the SWNT. The H-site, i.e., above the cen-
PACS number(s): 73.22.-f, 68.43.Bc, 68.43.Fg, 73.20.Hb

ter of hexagon formed by C-C bonds, is found to be energetically most favorable site with a binding energy of $E_{b}$ $=2.2 \mathrm{eV}$ for the magnetic ground state. The average $\mathrm{C}-\mathrm{Ti}$ distance, $\bar{d}_{\mathrm{C}-\mathrm{Ti}}$ has been found to be $2.2 \AA$. At the internal $\mathrm{H}$ site, the bonding is stronger and the binding energy is $E_{b}$ $=2.5 \mathrm{eV}$, the average bond distance $\bar{d}_{\mathrm{C}-\mathrm{Ti}}=2.3 \AA .{ }^{11}$ The magnetic moment of the individual Ti absorbed $(8,0)$ SWNT is calculated to be $\mu=2.2 \mu_{B}$ (Bohr magneton). Ti $3 d$ orbitals play a crucial role in the bonding and electrons are transferred from Ti to SWNT. ${ }^{11,12}$

A strong Ti-SWNT chemical interaction is responsible

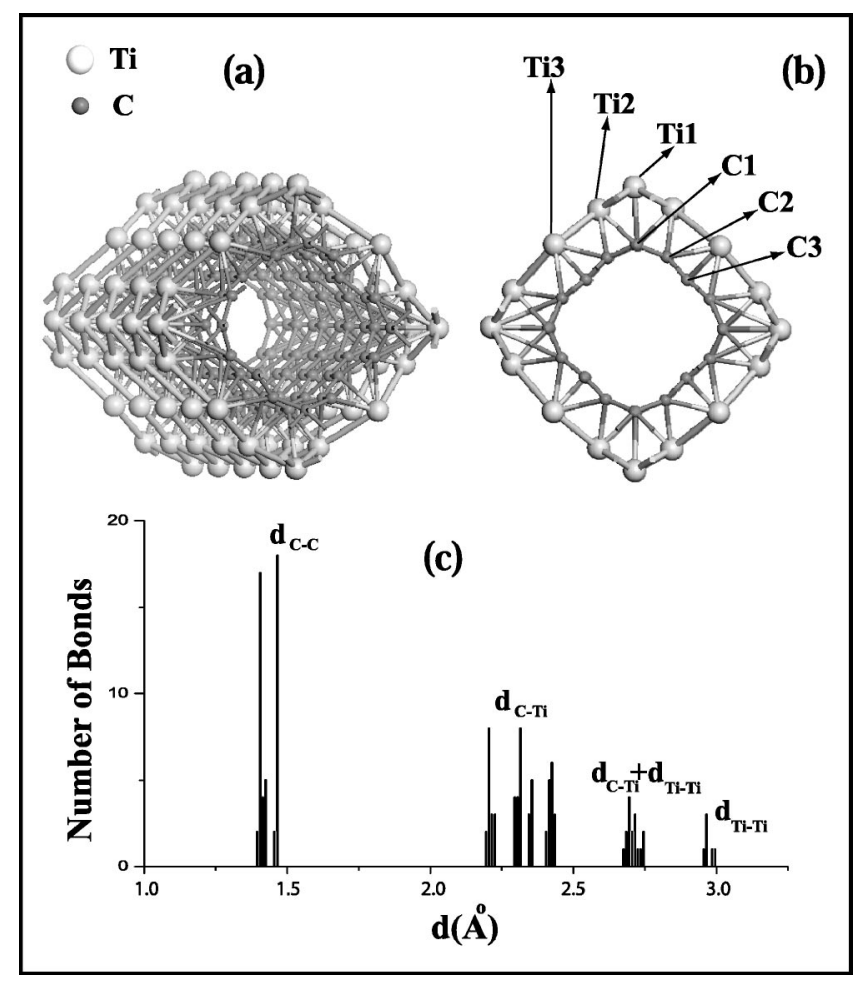

FIG. 1. (a) Fully optimized atomic structure of Ti-covered $(8,0)$ SWNT. (b) The cross section with different types of $\mathrm{C}$ atoms (identified as C1, C2, and C3) and adsorbed Ti atoms (Ti1, Ti2, and Ti3). Dark-small and light-large circles indicate $\mathrm{C}$ and $\mathrm{Ti}$ atoms, respectively. (c) Histograms show the variation of bond lengths of different carbon-carbon $\left(\bar{d}_{\mathrm{C}-\mathrm{C}}\right)$, carbon-Ti $\left(\bar{d}_{\mathrm{C}-\mathrm{Ti}}\right)$, and Ti-Ti $\left(\bar{d}_{\mathrm{Ti}-\mathrm{Ti}}\right)$ bonds. 

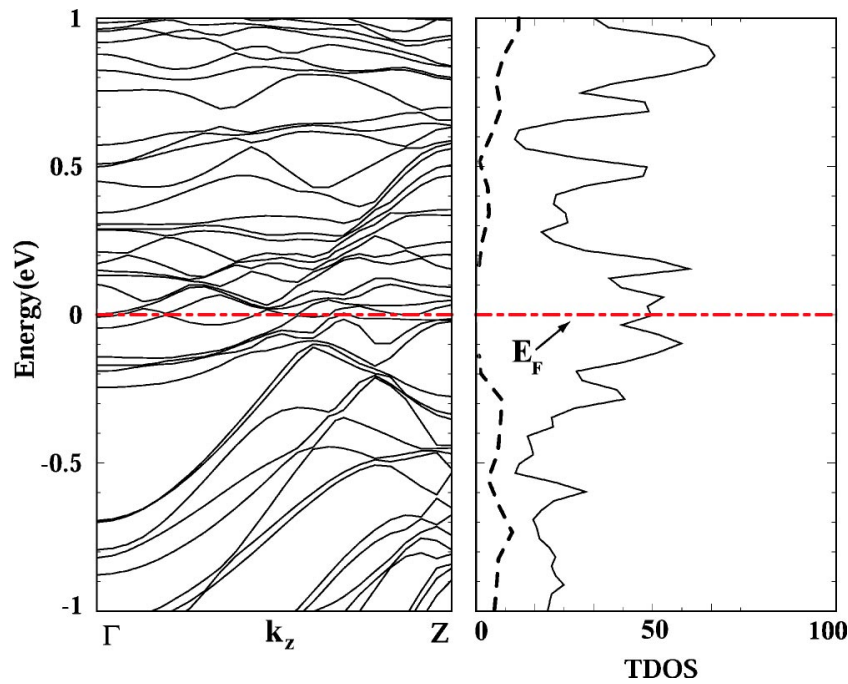

FIG. 2. (Color online) (a) Electronic energy band structure of a Ti-covered $(8,0)$ SWNT. (b) The total density of states (TDOS). TDOS of bare $(8,0)$ tube is shown by dashed lines. Zero of energy is taken at the Fermi level.

for the continuous coating. Here, the Ti coverage of $(8,0)$ SWNT has been analyzed first by attaching Ti atoms at all $\mathrm{H}$ sites in the unit cell, and subsequently by optimizing the atomic structure and the lattice constant $c$. The relaxation of the SWNT lattice was crucial in obtaining stable structures; frozen lattice constant has led to instabilities. The average binding energy, $\quad \bar{E}_{b}=\left(16 E_{T}[\mathrm{Ti}]+E_{T}[\mathrm{SWNT}]-E_{T}[16 \mathrm{Ti}\right.$ $+\mathrm{SWNT}]$ )/16, has been found (in terms of the total energies of individual Ti atom, optimized bare SWNT, and Ti-covered $\mathrm{SWNT}$ ) to be $4.3 \mathrm{eV}$. Apparently, owing to the Ti-Ti coupling, $\bar{E}_{b}$ comes out much higher than the binding energy of the adsorbed single Ti atom. For the same reason the charge transfer from $\mathrm{Ti}$ to $\mathrm{C}$ has decreased to $\sim 0.3$ electrons, and $\bar{d}_{\text {C-Ti }}$ increased to $\sim 2.5 \AA$.

The optimized atomic structure shown in Fig. 1 depicts an interesting feature of the Ti-covered SWNT. Atoms have rearranged in a quasi-1D "crystalline" structure and formed a squarelike cross section. We distinguish three specific $\mathrm{C}$ atoms (identified as $\mathrm{C} 1, \mathrm{C} 2$, and $\mathrm{C} 3$ ) and three Ti atoms (Ti1, Ti2, and Ti3) depending on their different bonding geometry. The $\mathrm{C} 1$ and Til atoms located at the corner of square are at the high curvature site, while $\mathrm{C} 3$ and Ti3 are at the flat region. In spite of the periodic arrangement of adsorbed $\mathrm{Ti}$ and underlying $\mathrm{C}$ atoms, the $\mathrm{Ti}-\mathrm{Ti}, \mathrm{Ti}-\mathrm{C}$, and $\mathrm{C}-\mathrm{C}$ bond distances show some dispersion depending on their location. The histogram in Fig. 1 identifies different types of bonds at different places.

The energy band structure and the total density of states of the Ti-covered $(8,0)$ SWNT are presented in Fig. 2. The band structure of the bare semiconducting $(8,0)$ SWNT has changed dramatically having several bands crossing the Fermi level. Accordingly, the Ti-covered SWNT becomes a good conductor with high density of states at the Fermi level, $\mathcal{D}\left(E_{F}\right)$. The current associated with the electron transport can be given by a Landauer type expression, ${ }^{13}$

$$
I\left(V_{b}\right)=\frac{2 e^{2}}{h} \int_{\mu_{r}}^{\mu_{l}} d E\left(f_{l}-f_{r}\right) \mathcal{T}\left(E, V_{b}\right) d E
$$

in terms of the bias voltage $V_{b}$; the Fermi distribution function of left and right electrodes $f_{l}$ and $f_{r}$, and their chemical potentials $\mu_{l}$ and $\mu_{r}$. Electron scattering in the contacts is crucial for the calculation of conductance. Therefore, the calculation of quantum conductance $\mathbf{G}$ of an interconnect between two electrodes requires detailed description of the contacts and phonon spectrum at the operation temperature. Here, since we are concerned only with the nanowire, we infer $\mathbf{G}$ from an ideal Ti-covered SWNT. Under these circumstances, the mean-free path of electrons $l_{m}$ becomes infinite at $T=0$, and the electronic transport occurs ballistically and coherently. This situation has been treated as an ideal 1D constriction, where the electrons are confined in the

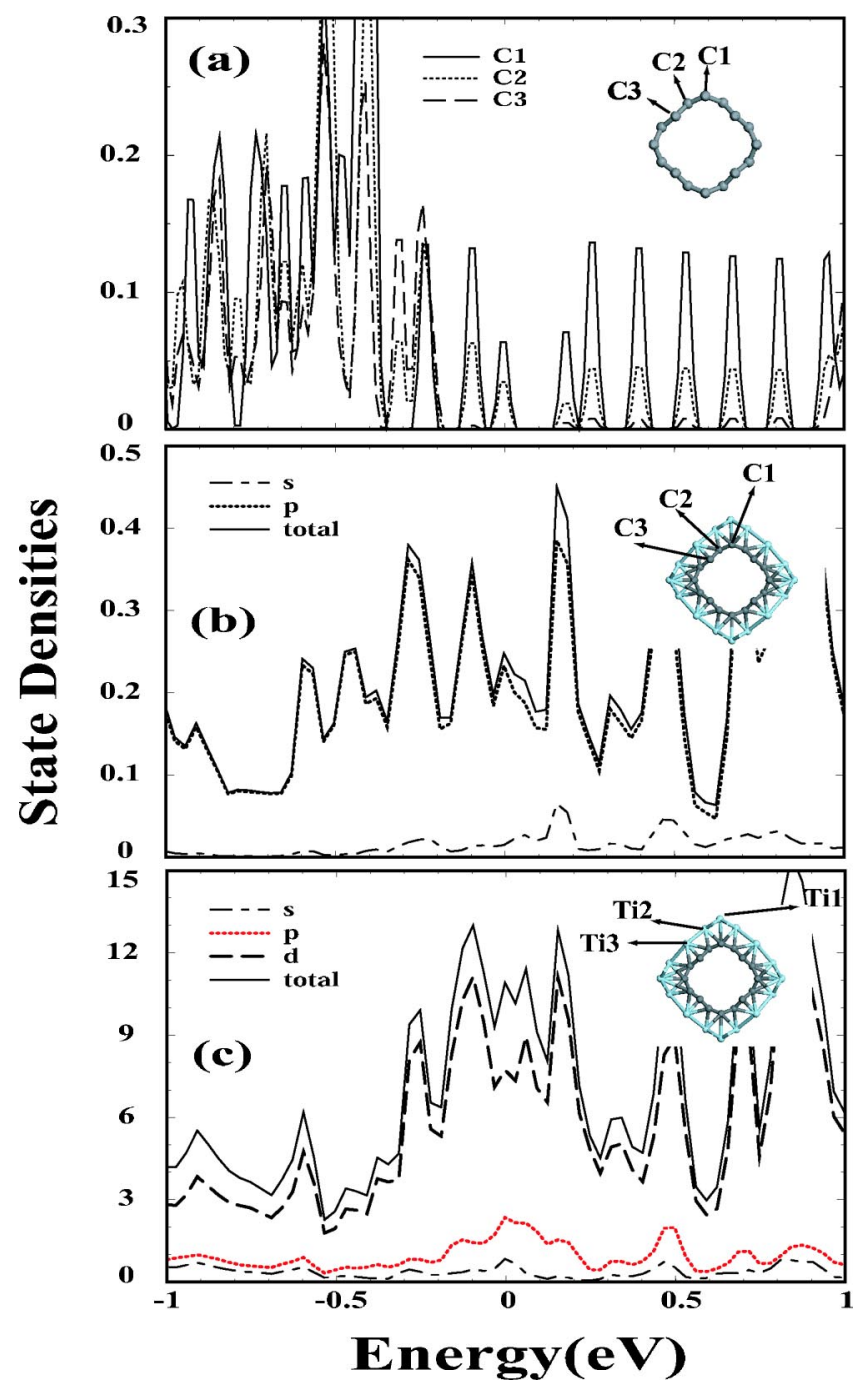

FIG. 3. (Color online) Calculated state densities. (a) Local density of states (LDOS) on C1, C2, C3 atoms of carbon nanotube which has the same atomic configuration and squarelike cross section as the carbon nanotube covered by Ti as shown in Fig. 1 (see inset). (b) LDOS on the carbon atoms of the Ti-covered SWNT (i.e., $\mathrm{C} 1+\mathrm{C} 2+\mathrm{C} 3$ ). (c) LDOS calculated on the Ti atoms of the Ticovered SWNT (i.e., Ti1 + Ti2 + Ti3). Partial density of states of $s$, $p$, and $d$ orbitals are also shown. 


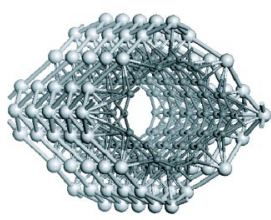

$(8,0)+\mathrm{Ti}$

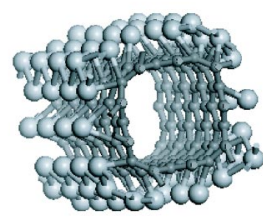

$(9,0)+\mathrm{Ti}$
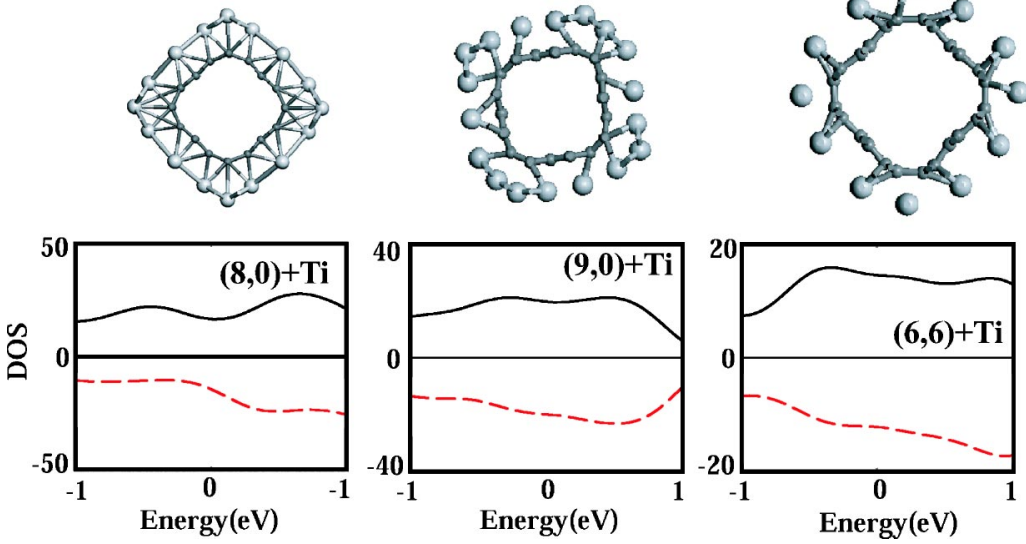

FIG. 4. (Color online) Optimized atomic structure of $(8,0)$ and $(9,0)$ zigzag, and $(6,6)$ armchair SWNT which are uniform covered with Ti. Corresponding density of states for spin-up and spin-down electrons are shown. transversal direction, but propagate freely along the axis. ${ }^{5}$ The current is expressed as $I=\sum_{i} 2 \eta_{i} e v_{i}\left[\mathcal{D}_{i}\left(E_{F}+e V_{b}\right)\right.$ $\left.-\mathcal{D}_{i}\left(E_{F}\right)\right]$ where degeneracy, group velocity, and density of states of each subband crossing $E_{F}$ are given by $\eta_{i}, v_{i}, \mathcal{D}_{i}$, respectively. Since $\mathcal{D}_{i}\left(E_{F}+e V_{b}\right)-\mathcal{D}_{i}\left(E_{F}\right) \sim\left(e V_{b}\right) d \mathcal{D}_{i}(E) /$ $\left.d E\right|_{E_{F}}$ and $v_{i}=\left(h^{-1}\right) \partial \mathcal{D}_{i} /\left.\partial E\right|_{E_{F}}$, then $G=I / V_{b}=\Sigma_{i} 2 \eta_{i} e^{2} /$ $h$. Accordingly, each subband crossing the Fermi level is counted as $\eta_{i}$ current-carrying state for two spins with channel transmission $\mathcal{T}=1$. Then the maximum "ideal" conductance of defect-free Ti-covered ideal tube becomes $G$ $=2 e^{2} N_{b} / h$, where $N_{b}=\sum \eta_{i}$. Calculated conductance is four times higher than that of bare metallic armchair tube. High $\mathcal{D}\left(E_{F}\right)$ in Fig. 2 justifies this result.

In reality, $\mathcal{T}(E)$ in Eq. (1) is reduced due to scattering of carriers from the abrupt change of cross sections and irregularities at the contacts to electrodes and from the imperfections, impurities, and electron-phonon scattering in the tube by itself. We note that the regular structure shown in Fig. 1 may occur under idealized conditions; normally irregularities are unavoidable, in particular for a thick Ti coating. While the channel transmission is decreased in the thick but inhomogeneous Ti coating, $\mathbf{G}$ is expected to be still high owing to the new conductance channels opened at $E_{F}$. Based on these arguments and in view of the high $\mathcal{D}\left(E_{F}\right)$ in Fig. 2, the conductance of a Ti coated tube can be several $2 e^{2} / h$.

Any defect in 1D system gives rise to the localization of current transporting states which is characterized by the localization length $\xi$. While $\xi \sim l_{m}$ for a strictly 1D wire, $\xi$ $\sim l_{m} d / \lambda_{F}$ for a 1D stripe and $\xi \sim l_{m} d^{2} / \lambda_{F}^{2}$ for a wire with width or diameter $d$ and Fermi wavelength, $\lambda_{F}=h / m v_{F} \cdot{ }^{14}$ We expect that for the present Ti-covered SWNT $\xi$ $\sim l_{m}\left(d / \lambda_{F}\right)^{\alpha}$ with $1<\alpha<2$. Then the net resistance of ( $\mathrm{Ti}$ + SWNT) wire having length $L$ between two contacts and including contact resistance $R_{c}$ and localization effect can be given by $R=R_{c}+h e^{L / \xi} / 2 e^{2}$. A very crude estimation yields $\xi$, which is much larger than a typical $L$ for interconnects in nanoelectronics.
The origin of metallicity is the next question we will address. First, let us consider the nanotube having the same atomic configuration, hence the same squarelike cross section as in Fig. 1, but depleted from all adsorbed $\mathrm{Ti}$ atoms. The local densities of states (LDOS) at C1, C2, and C3 carbon atoms in Fig. 3(a) clarify whether such a deformed SWNT continues to be semiconducting. For the atom C3, which is located at the center of the edge of square, i.e., at the flat region of the tube the state density vanishes at $E_{F}$. In contrast, as one approaches the corner, LDOS at $E_{F}$ increases, and eventually at $\mathrm{C} 1$ (i.e., the atom at the corner of the square) has the highest density. This situation implies that the square nanotube by itself (without Ti) can be viewed as four metal strips passing through its four corners, and four semiconductors at the flat edges. The metallization is induced by the singlet conduction band that crossed the Fermi level due to enhanced $\pi^{*}-\sigma^{*}$ hybridization at the corner region in Figs. 3(a) and 3(b). ${ }^{15-17}$ In Fig. 3(c), the LDOS and orbital projected LDOS calculated at $\mathrm{Ti}$ atoms have high state density at $E_{F}$ due to the states derived mainly from $\mathrm{Ti}$ $3 d$ orbitals. Accordingly, the main contribution to the high $\mathcal{D}\left(E_{F}\right)$ in Fig. 2 is due to adsorbed Ti atoms, but the underlying carbon tube itself has some contribution.

Uniform coverage of $\mathrm{Ti}$ on the SWNT is crucial for the future technological applications. Ti can be used as a buffer layer to form uniform coating of good conductors, such as $\mathrm{Au}, \mathrm{Cu}$, on the SWNT, ${ }^{11}$ since these atoms have low binding energy (0.5 and $0.7 \mathrm{eV}$, respectively). Strong Ti-SWNT interaction can be utilized to bond or to connect individual SWNT's in order to form $T, Y$ and cross junctions or grids. It appears that fabrication of photonic band gap materials or nanowave guides based on SWNT's may not be a mere speculation. Earlier, it has been shown that quantum structures can be realized on a single s-SWNT through band-gap modulation either by modulating radial deformation or by modulating adsorption of hydrogen atoms. ${ }^{16,18}$ These quantum structures can be connected to the electrodes through 
their both ends which are metallized by $\mathrm{Ti}$ coverage. This way one can fabricate an electronic nanodevice on a single SWNT, such as a Schottky barrier diode or a resonant tunneling device.

The uniform Ti coverage of a SWNT may be of interest in a completely different field of research. Individual $\mathrm{Fe}$ atom is weakly bound on a SWNT, but it has a magnetic ground state. The binding energy and the magnetic moment have been calculated to be $E_{b}=0.8 \mathrm{eV}, \mu \approx 2 \mu_{B}$, respectively. However, our calculations have revealed that $\mathrm{Fe}$ atoms cannot form a uniform and continuous coverage; rather they are accumulated into small clusters attached to the surface of the SWNT. Alternatively, uniform coating of $\mathrm{Fe}$ can be realized over the Ti-covered SWNT and hence nanomagnets can be generated. The exchange interaction and the ferromagnetism of these quasi-1D nanomagnets would be an important subject of study. The individual $\mathrm{Cr}$ and $\mathrm{Mn}$ adsorbed on the $(8,0)$ tube have also low binding energy $\left(E_{b} \sim 0.4 \mathrm{eV}\right)$, but high magnetic moment $\left(\mu=5.17\right.$ and $5.49 \mu_{B}$, respectively). ${ }^{11}$ Similar to the case of Fe, magnetic nanostructures can be obtained when these atoms cover or decorate the Ti-covered SWNT. This way, the band structures of these magnetic nanostructures can be engineered for desired spin-dependent electron transport. Interestingly, the SR total energy of Ticovered $(8,0)$ tube is found to be $\sim 0.6 \mathrm{eV} /$ cell lower than $\mathrm{SU}$ total energy. Hence Ti-covered $(8,0)$ tube shown in Fig. 1 has magnetic ground state with calculated magnetic moment of $\sim 15.3 \mu_{B}$. The magnetization and hysteresis loops of iron nanoparticles partially encapsulated at the tips and inside of aligned carbon nanotubes have been demonstrated by recent experimental works. ${ }^{19}$ Through $a b$ initio calculations it has been shown that SWNT's filled or coated with transition- metal elements can exhibit substantial spin polarization. ${ }^{20}$

Finally, we demonstrated that the uniform coverage of Ti resulting in a regular atomic structure occurs also for SWNT's with different radius and chirality. Figure 4 shows the optimized atomic structure of Ti-covered $(9,0)$ zigzag and $(6,6)$ armchair tubes. The former bare tube has a very small band gap of $0.09 \mathrm{eV}$, radius of $3.6 \AA$, and odd number of $\mathrm{C}$ atoms on the circumference. The latter tube is a metal and has a radius of $4.1 \AA$ and chiral angle of $30^{\circ}$ when it is free of Ti. However, both tubes become metal with high $\mathcal{D}\left(E_{F}\right)$ and have a magnetic ground state upon coverage with Ti. The calculated magnetic moments are $13.7 \mu_{B}$ and $9.5 \mu_{B}$ for $(9,0)$ and $(6,6)$ SWNT's, respectively. Sharp corners of the squarelike cross section in Fig. 1 start to be flattened and change to polygonal form for the Ti-covered $(6,6)$ SWNT.

In conclusion, we showed that $\mathrm{Ti}$ atoms can form a uniform coverage on SWNT's. Upon Ti coverage the Ti + SWNT nanowire undergoes three major changes.

(i) Depending on the radius and the chirality the circular cross section changes to either squarelike or polygonal form. However, these forms may be modified at higher Ti coverage.

(ii) It becomes a quasi-1D metal with high state density at $E_{F}$. Owing to the uniform and periodic atomic structure, the several states at $E_{F}$ contribute to quantum ballistic transport with high transmission probability and hence high conductance.

(iii) Ti-covered SWNT's have a magnetic ground state with net magnetic moment.

This work was partially supported by the National Science Foundation under Grant No. INT01-15021 and TÜBITAK under Grant No. TBAG-U/13(101T010). S.C. thanks Professor I.O Kulik for discussions.
${ }^{1}$ H. Ohnishi, Y. Kondo, and K. Takayanagi, Nature (London) 395, 783 (1998).

${ }^{2}$ A.I. Yanson, G.R. Bollinger, H.E. Brom, N. Agraï, and J.M. Ruitenbeek, Nature (London) 395, 783 (1998).

${ }^{3}$ N. Agrait, G. Rubio, and S. Vieira, Phys. Rev. Lett. 74, 3995 (1995)

${ }^{4}$ S. Ciraci and E. Tekman, Phys. Rev. B 40, 11969 (1989); E. Tekman and S. Ciraci, ibid. 43, 7145 (1991); H. Mehrez, S. Ciraci, A. Buldum, and I.P. Batra, ibid. 55, R1981 (1997).

${ }^{5}$ S. Ciraci, A. Buldum, and I.P. Batra, J. Phys.: Condens. Matter 13, R537 (2001).

${ }^{6}$ A. Buldum, S. Ciraci, and C.Y. Fong, J. Phys.: Condens. Matter 12, 3349 (2000); A. Buldum, D.M. Leitner, and S. Ciraci, Europhys. Lett. 47, 208 (1999); A. Ozpineci and S. Ciraci, Phys. Rev. B 63, 125415 (2001).

${ }^{7}$ H. Dai, E.W. Wong, Y.Z. Lu, S. Fan, and C.M. Lieber, Nature (London) 375, 769 (1995); W.Q. Han, S.S. Fan, Q.Q. Li, and Y.D. Hu, Science 277, 1287 (1997).

${ }^{8}$ Y. Zhang and H. Dai, Appl. Phys. Lett. 77, 3015 (2000); Y. Zhang, N.W. Franklin, R.J. Chen, and H. Dai, Chem. Phys. Lett. 331, 35 (2000).

${ }^{9}$ C. Zhou, J. Kong, and H. Dai, Phys. Rev. Lett. 84, 5604 (2000).

${ }^{10}$ J.P. Perdew, J.A. Chevary, S.H. Vosko, K.A. Jackson, M.R. Pederson, D.J. Singh, and C. Fiolhais, Phys. Rev. B 46, 6671 (1992).
${ }^{11}$ E. Durgun, S. Dag, V.K. Bagci, O. Gülseren, T. Yildirim, and S. Ciraci, Phys. Rev. B 67, 201401(R) (2003); E. Durgun, S. Dag, S. Ciraci, and O. Gülseren, J. Phys. Chem. B 108, 575 (2004).

${ }^{12}$ C.-K. Yang, J. Zhao, and J.P. Lu, Phys. Rev. B 66, 041403 (2002).

${ }^{13}$ C.C. Kaun, B. Larade, H. Mehrez, J. Taylor, and H. Guo, Phys. Rev. B 65, 205416 (2002).

${ }^{14}$ B. L. Altshuler and A. G. Aronov, in Electron-Electron Interaction in Disordered Systems, edited by A. L. Efros and M. Pollak (Elsevier, Amsterdam, 1985).

${ }^{15}$ X. Blase, L.X. Benedict, E.L. Shirley, and S.G. Louie, Phys. Rev. Lett. 72, 1878 (1994).

${ }^{16}$ C. Kilic, S. Ciraci, O. Gülseren, and T. Yildirim, Phys. Rev. B 62, 16345 (2000).

${ }^{17}$ O. Gülseren, T. Yildirim, and S. Ciraci, Phys. Rev. Lett. 87, $116802(2001)$

${ }^{18}$ O. Gülseren, T. Yildirim, and S. Ciraci, Phys. Rev. B 68, 115419 (2003).

${ }^{19}$ X.X. Zhang, G.H. Wen, S. Huang, L. Dai, R. Gao, and Z.L. Weng, J. Magn. Magn. Mater. 231, L9 (2001); B.C. Satishkumar, A. Govindaraj, P.V. Vanitha, A.K. Raychaudhuri, and C.N.R. Rao, Chem. Phys. Lett. 362, 301 (2002).

${ }^{20}$ C.-K. Yang, J. Zhao, and J.P. Lu, Phys. Rev. Lett. 90, 257203 (2003). 